Revue des patrimoines

\title{
Particularités des moulins hydrauliques d'Alsace Bossue (Bas-Rhin)
}

\section{Franck Schwarz}

\section{(2) OpenEdition}

\section{Journals}

Édition électronique

URL : http://journals.openedition.org/insitu/2893

DOI : 10.4000/insitu.2893

ISSN : $1630-7305$

\section{Éditeur}

Ministère de la culture

Référence électronique

Franck Schwarz, « Particularités des moulins hydrauliques d'Alsace Bossue (Bas-Rhin) », In Situ [En ligne], 8 | 2007, mis en ligne le 01 mars 2007, consulté le 19 avril 2019. URL : http:// journals.openedition.org/insitu/2893 ; DOI : 10.4000/insitu.2893

Ce document a été généré automatiquement le 19 avril 2019

\section{(c) $($ i) $(9)$}

In Situ Revues des patrimoines est mis à disposition selon les termes de la licence Creative Commons Attribution - Pas d'Utilisation Commerciale - Pas de Modification 4.0 International. 


\title{
Particularités des moulins hydrauliques d'Alsace Bossue (Bas-Rhin)
}

\author{
Franck Schwarz
}

1 Située au nord-ouest du département du Bas-Rhin, l'Alsace Bossue apparaît comme une enclave alsacienne en terre lorraine. Ce territoire ${ }^{1}$ à vocation essentiellement rurale bénéficie d'un réseau hydrographique dense qui a favorisé l'établissement de moulins hydrauliques dès le XIe siècle. Ce corpus a fait l'objet d'une enquête thématique ${ }^{2}$ qui a permis de localiser 37 unités productives à l'échelle des deux cantons inventoriés (SarreUnion et Drulingen). Celles-ci présentent des particularités marquées qui découlent de la nature du territoire et de son long enclavement.

2 Nous nous attacherons à développer cette singularité qui s'articule autour d'une concentration tardive et moins affirmée qu'à proximité des grands centres urbains, d'une introduction plus lente de la mécanisation des chaînes de fabrication et de la permanence d'une architecture peu différenciée qui en est le résultat, enfin d'une fidélité indéfectible à l'énergie hydraulique.

\section{Une concentration inéluctable mais tardive}

3 A la fin du XIXe siècle, le domaine de la meunerie est marqué par un contexte de concentration et de concurrence exacerbée. Les phases successives de mécanisation donnent naissance aux minoteries. Les moulins ruraux, pratiquant la mouture à façon, ne peuvent s'adapter et disparaissent inexorablement. Ce constat est dressé par l'ensemble des observateurs régionaux. Charles Grad relève ainsi, en 1879, que "les moulins de l'ancien système (sont) fatalement condamnés à disparaître, ils ne servent guère plus qu'à fabriquer la farine de ménage des cultivateurs "3. L'Alsace Bossue n'échappe pas à cette évolution même si le processus est plus tardif et plus nuancé qu'aux abords des grandes agglomérations comme Strasbourg. L'éloignement de ces zones urbaines où se concentrent désormais les grandes minoteries, affranchies des contraintes de l'énergie hydraulique et à proximité des grandes voies de circulation, préserve un marché local et 
offre un certain répit à ces moulins. L'évolution est toutefois inéluctable. Les établissements décidés à survivre sont contraints de s'équiper de broyeurs à cylindres pour accroître leur rendement et délivrer une farine plus blanche et d'un plus grand degré de finesse. Pour les autres, le déclin est amorcé. Ils disparaissent en grand nombre au cours de la dernière décennie du XIXe siècle. Les moulins d'Altwiller ${ }^{4}$ et de Bettwiller (Aumuehle) suspendent leur activité en 1894. Le moulin de Keskastel est reconverti en centrale hydroélectrique en 1901. Valentin Pelsy observe aussi, en 1896, une "forte diminution du nombre de nos moulins actifs " ${ }^{5}$. (fig. $\mathrm{n}^{\circ} 1$ )

Figure 1

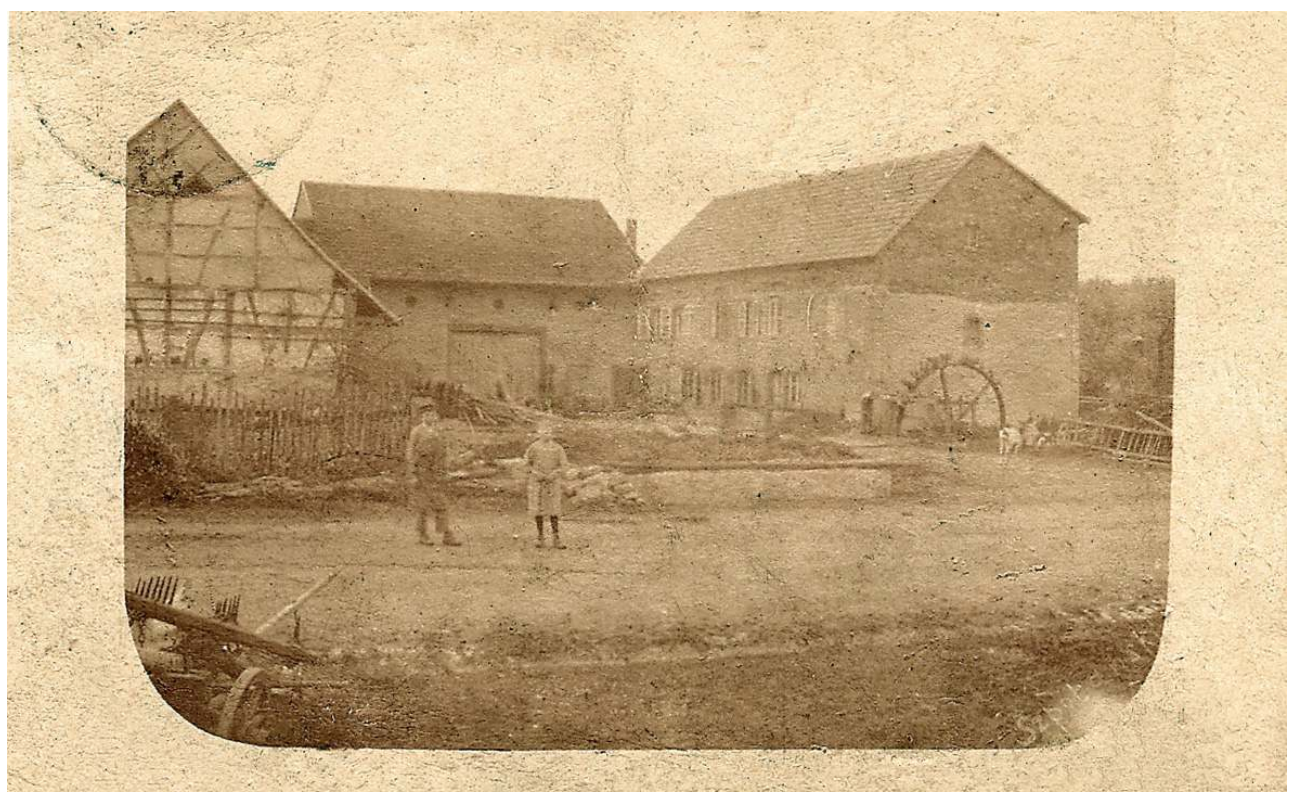

Le moulin d'Altwiller, dont l'activité a cessé en 1894 (collection particulière). Repro. Inv. F. Schwarz

(c) Inventaire général, ADAGP, 2005

4 Au plan local, le processus de concentration donnant naissance à des établissements de grande ampleur est circonscrit aux sites de Wolfskirchen et de Drulingen. De petits moulins ruraux qu'ils étaient, ils se développent et se modernisent jusqu'à acquérir une dimension industrielle. Produisant en grande quantité, ils exportent leur farine au moyen de camions sur un marché qui excède les limites cantonales ; ils se caractérisent aussi par un accroissement progressif de leur espace productif et la mise en œuvre des innovations qui ponctuent l'histoire de la meunerie. (fig. $n^{\circ} 2, n^{\circ} 3$ ) 
Figure 2

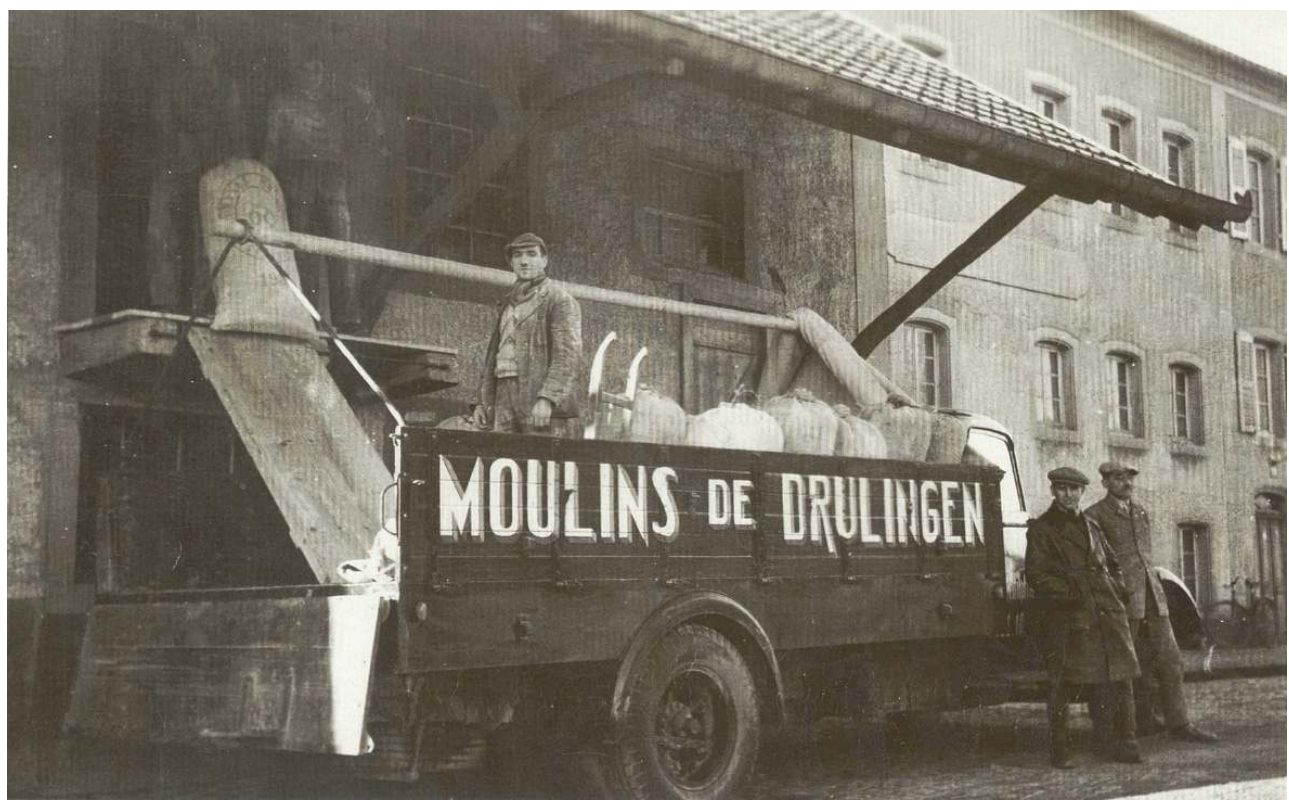

Chargement des sacs de farine au moulin de Drulingen (collection particulière). Repro. Inv. F. Schwarz (c) Inventaire général, ADAGP, 2006

Figure 3

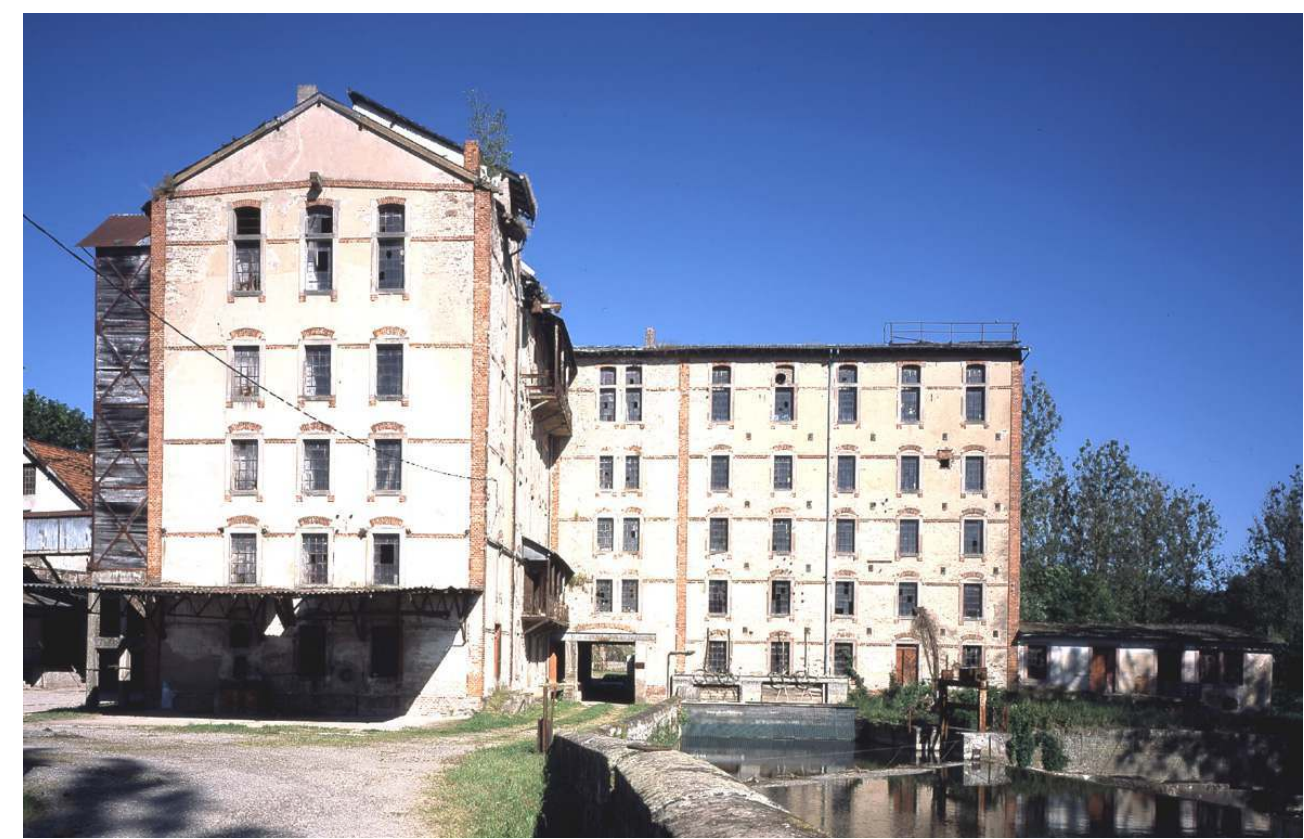

Elévation méridionale de la minoterie Schlumberger de Wolfskirchen. Phot. Inv. J. Raimbault (c) Inventaire général, ADAGP, 2005

En 1938, l'Alsace Bossue ne compte plus que 15 moulins en activité, dont ceux de Wolfskirchen et de Drulingen. En moins de soixante ans, le territoire a perdu près des deux tiers de ses établissements. Ne subsistent que ceux qui ont su s'adapter et adopter les nouveaux procédés de mouture. Les autres connaissent des destins divers. Si certains changent d'affectation industrielle, ce phénomène est cependant moins marqué que dans 
d'autres régions en raison de son caractère tardif. Lorsque les moulins d'Alsace Bossue cèdent le pas, à la charnière des XIXe et XXe siècles, l'industrie appuie son développement sur l'énergie thermique. Dans les vallées vosgiennes, en revanche, l'installation d'usines textiles au cours de la première moitié du XIXe siècle est conditionnée par la présence d'un cours d'eau : nombreux sont alors les anciens moulins réinvestis par l'industrie cotonnière.

6 Les reconversions sont fonction de facteurs locaux ou conjoncturels. A Waldhambach, la présence de nombreuses forêts conduit le meunier de la Kuppertsmuehle à se réorienter vers le sciage du bois. A Keskastel, l'implantation de la firme Marmor, spécialisée dans la taille du marbre, du granite et de la pierre, nécessite l'aménagement d'une usine de production d'énergie. L'entreprise jette son dévolu sur le moulin de la commune et l'équipe de deux turbines. Enfin à Sarrewerden, les deux moulins qui se dressent à proximité des anciens remparts sont acquis en 1910 et 1911 par Joseph Hirtz, propriétaire d'une verrerie en Lorraine. Cherchant à se développer, il réinvestit les espaces productifs et en accroît la surface par la construction d'une halle au cours des années 1960 . (fig. nº 4 , $\left.n^{\circ} 5\right)$

Figure 4

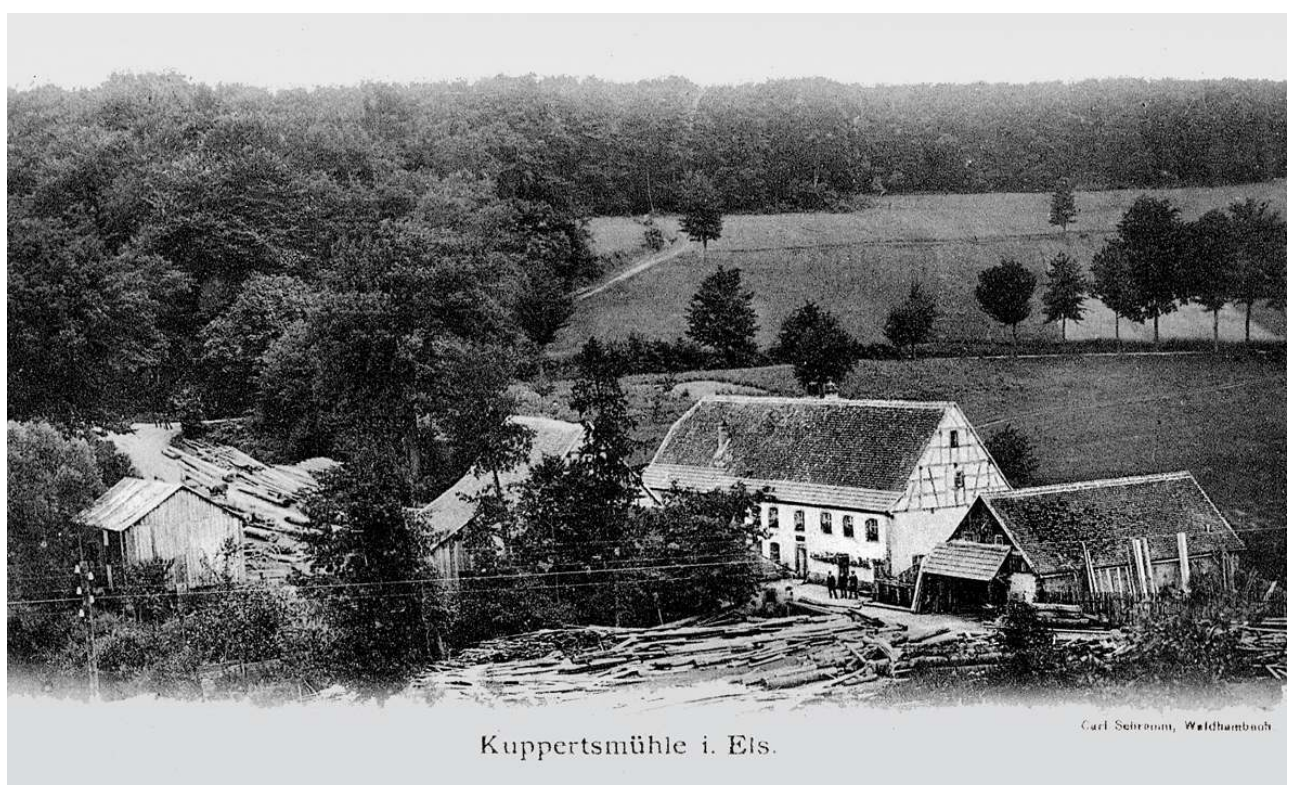

La scierie Kuppertsmuehle à Waldhambach vers 1900 (collection particulière). Repro. Inv. F. Schwarz (c) Inventaire général, ADAGP, 2005 
Figure 5

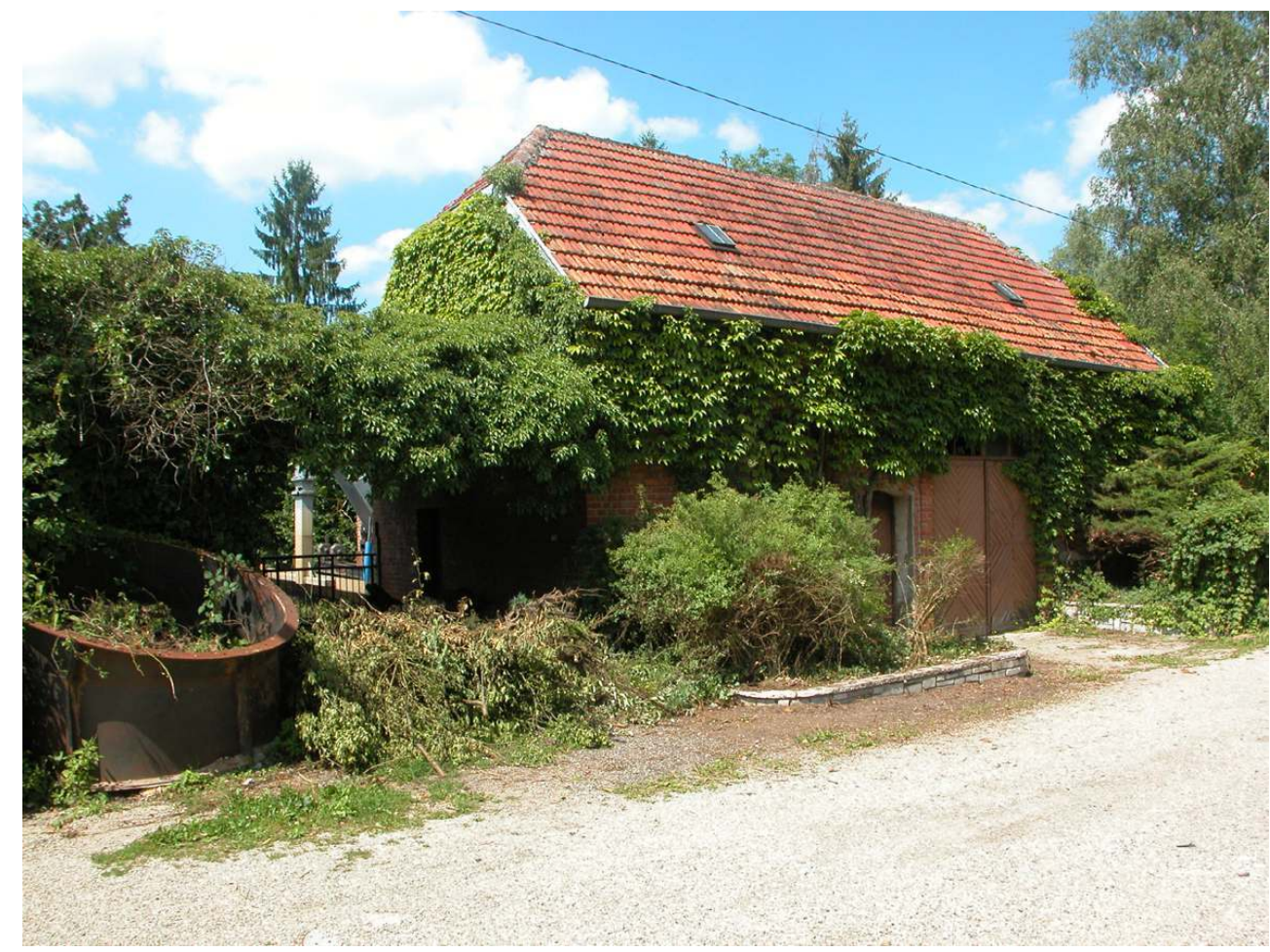

La centrale hydroélectrique de Keskastel. Phot. Inv. F. Schwarz

(C) Inventaire général, ADAGP, 2005

7 La majeure partie des moulins d'Alsace Bossue s'est vue transformer en habitation. Ce constat vaut tant pour les anciens moulins qui disparaissent à la fin du XIXe siècle en raison de leur archaïsme que pour les établissements qui poursuivent leur activité jusque dans les années 1970. Les vastes ateliers de fabrication, rendus nécessaires par la mécanisation de la mouture, sont cloisonnés. Les équipements techniques sont déposés et vendus. Les biefs de dérivation sont comblés. Toutefois, pour l'observateur averti, de nombreux signes trahissent la présence d'un ancien moulin. Les linteaux de portes sont souvent ornés de l'emblème de la profession. De même, l'implantation d'un bâtiment à proximité d'un cours d'eau n'est pas anodine. Les vestiges d'une meule disposés de manière ornementale constituent un autre témoignage du passé industriel du lieu. (fig. $n$ $\left.{ }^{\circ} 6\right)$ 


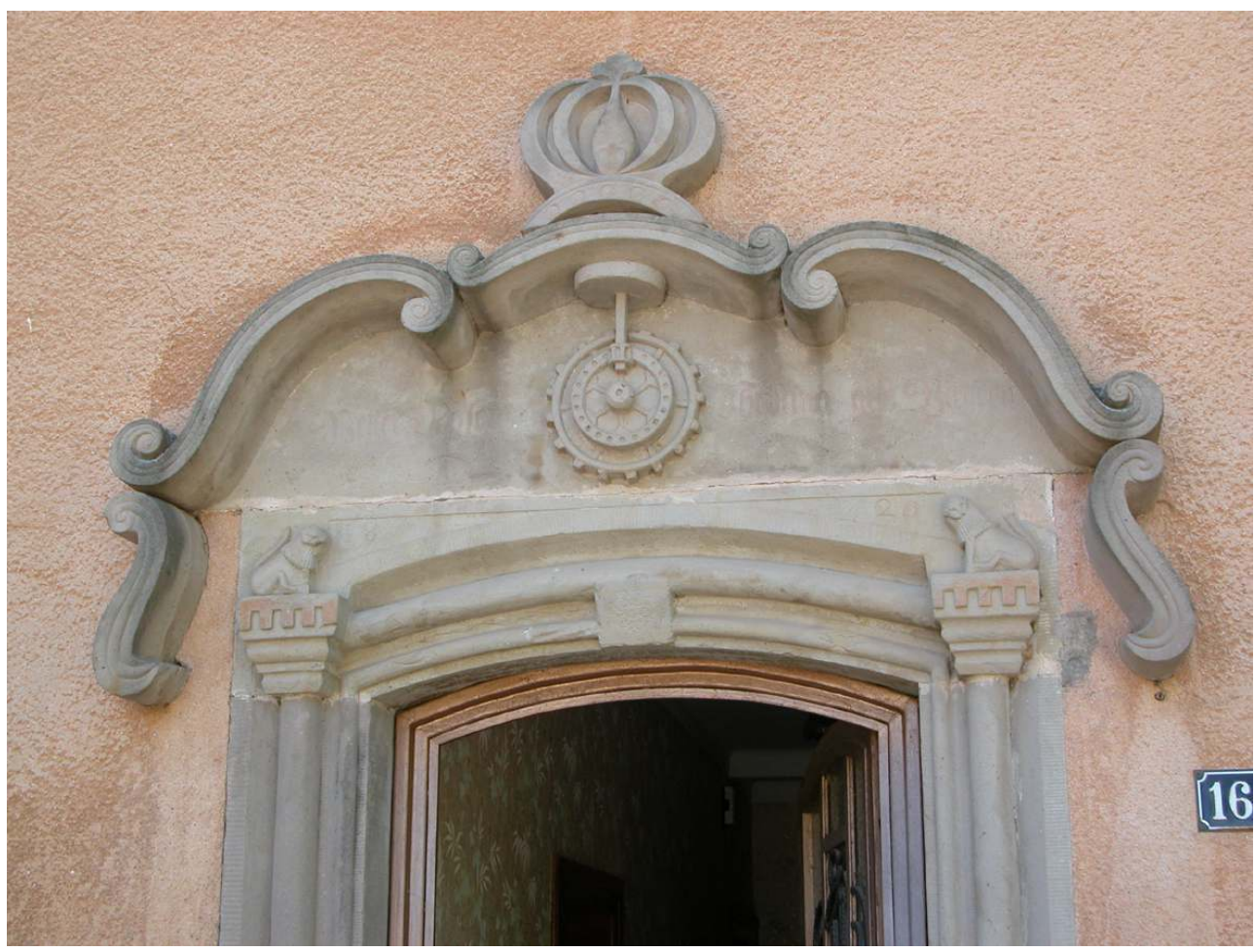

Linteau de porte du moulin de Schleifmuehle à Butten. On y distingue la roue hydraulique solidaire du rouet dont les alluchons engrènent les fuseaux de la lanterne, mettant ainsi la meule en mouvement. Phot. Inv. F. Schwarz

(C) Inventaire général, ADAGP, 2005

De nos jours, seuls deux établissements produisent encore de la farine en Alsace Bossue, à Harskirchen et Voellerdingen. Tous deux sont de petites unités de production qui ont su intégrer les évolutions techniques successives. Ils en ont progressivement adopté certains éléments, agrégeant de la sorte des équipements d'époques différentes. Le moulin de Voellerdingen actionne ainsi, au moyen de l'énergie électrique adoptée en 1971, des blutoirs installés en 1903. Ces derniers sont associés à des broyeurs à cylindres fournis en 1949 par les établissements Nehlig, Heuser et Cie. Le moulin de Willer à Harskirchen ${ }^{6}$ présente une hétérogénéité technique comparable. Les machines réservées au nettoyage du grain sont contemporaines de la première mécanisation du moulin, à la fin du XIXe siècle. Le broyeur à cylindres, toujours en activité, remonte au début des années 1920 tandis que le plansichter ${ }^{7}$ qui lui est associé a été installé en 1948. Ces deux établissements répondent à une demande locale qu'ils satisfont par de la vente directe et des livraisons quotidiennes. (fig. $n^{\circ} 7$ ) 
Figure 7

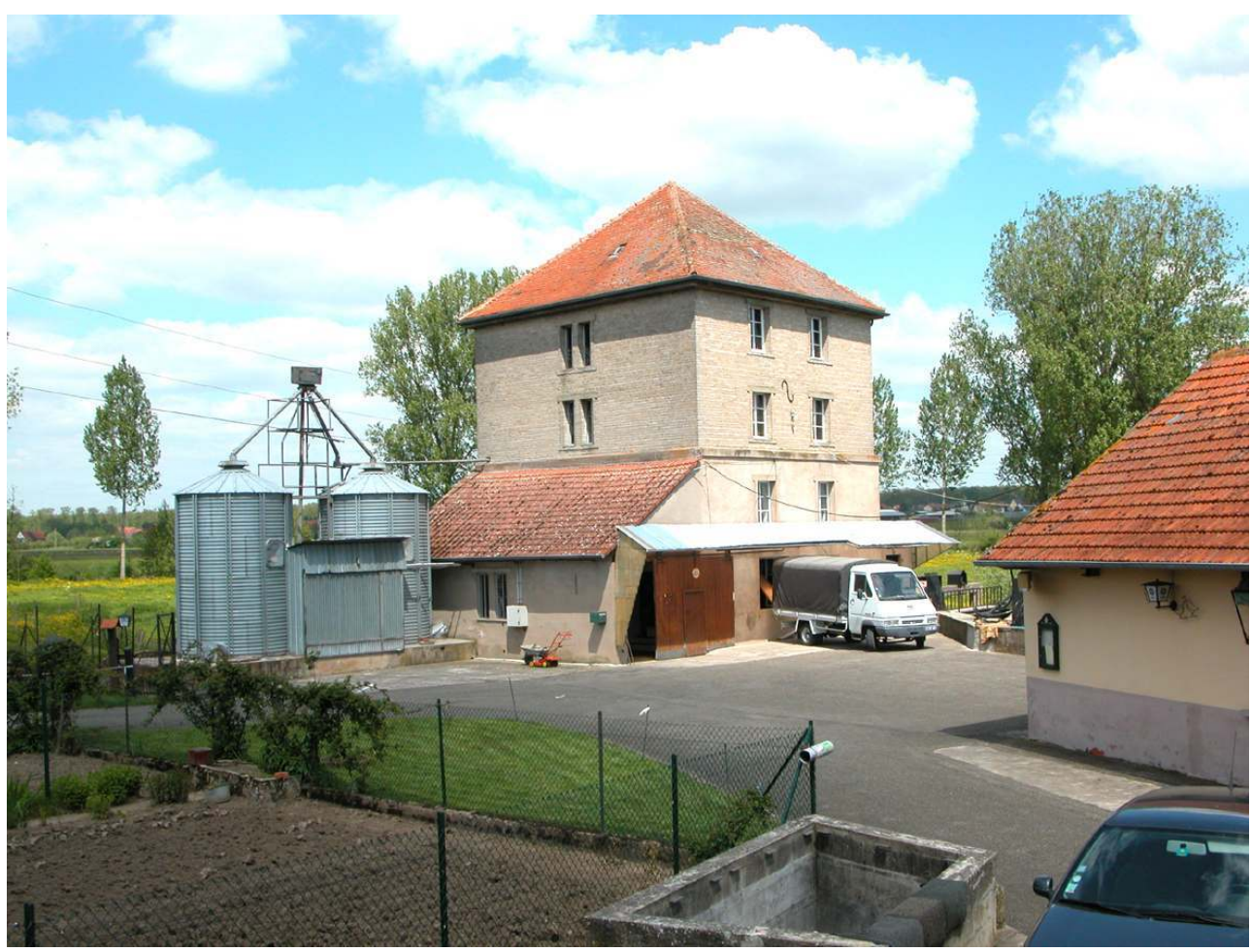

Le moulin de Willer à Harskirchen, toujours en activité. Phot. Inv. F. Schwarz

(C) Inventaire général, ADAGP, 2005

\section{Une mécanisation lente}

9 Tout au long du Moyen Age et de l'époque moderne, les moulins d'Alsace Bossue n'évoluent guère. Leur schéma technique est immuable. Une roue hydraulique entraîne dans sa rotation un rouet, solidaire de l'arbre moteur, dont les alluchons engrènent les fuseaux d'une lanterne qui transmet le mouvement à la meule. Les seules innovations marquantes apportées au cours des siècles consistent à rajouter une deuxième ou une troisième meule actionnée par sa roue propre. Ces meules en pierre sont importées majoritairement de Champagne mais également de La Ferté-sous-Jouarre en Seine-etMarne et de Cassel dans le Nord. Peu nombreux sont les établissements qui recourent aux carrières locales, celle de Wasselonne en particulier. (fig. $\mathrm{n}^{\circ} 8$ ) 


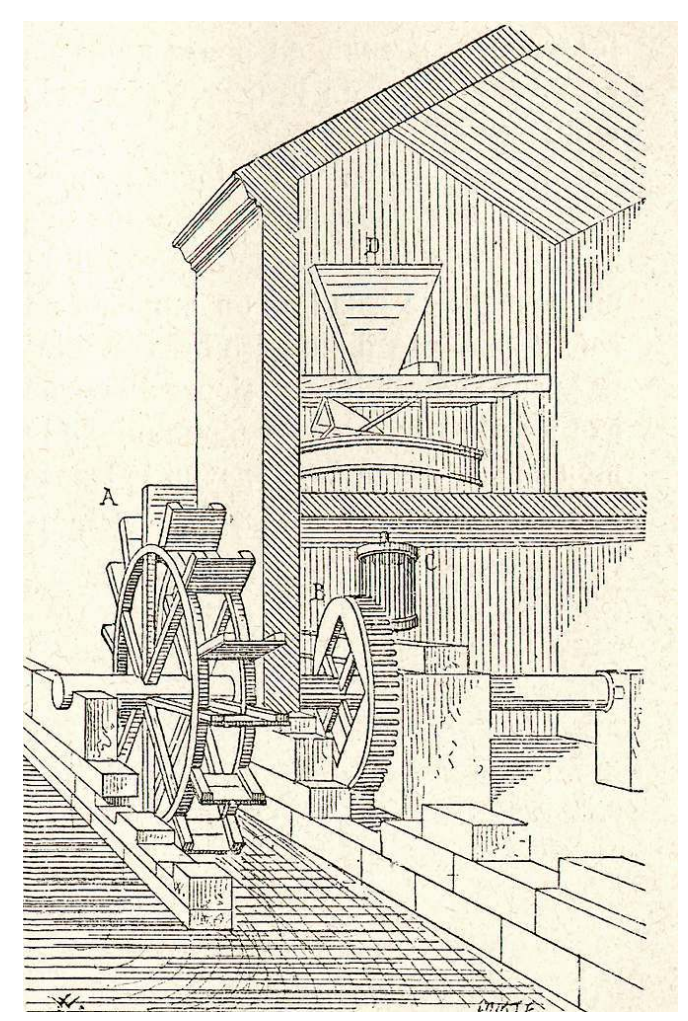

Rouet et lanterne transmettent le mouvement de la roue à la meule, publié dans P. Chabat, Dictionnaire des termes employés dans la construction, Paris, 1881. Repro. Inv. F. Schwarz

(C) Inventaire général, ADAGP, 2006

En dépit de quelques innovations à la veille de la Révolution, il faut attendre l'arrivée de nouveaux propriétaires à la tête des anciens moulins seigneuriaux pour que des améliorations conséquentes soient introduites. Les meuniers commencent ainsi à s'équiper en vue de nettoyer le grain préalablement à la mouture. Différents types de cylindres et de tarares munis d'un ventilateur et actionnés par l'énergie hydraulique font leur apparition. Par la suite, ils adoptent le bluteau pour tamiser la boulange brute au fur et à mesure de sa sortie des meules et délivrer ainsi une farine exempte de son. Ce tamis rudimentaire, formé d'un coffre dans lequel on tend un sac de toile, se maintient dans sa forme primitive durant la première moitié du XIXe siècle ${ }^{8}$. Pour autant les avancées techniques sont incessantes et connaissent leur apogée avec la mise au point de la “ mouture à l'américaine ” à laquelle Oliver Evans a attaché son nom9.

11 Cette nouvelle technique est porteuse de perfectionnements tant sur le plan du nettoyage des grains que du tamisage de la mouture ${ }^{10}$. Des blutoirs cylindriques, recouverts de soie et animés d'un mouvement rotatif se substituent aux anciens bluteaux. Les progrès portent également sur les mécanismes de transmission. Evans a l'idée de réunir plusieurs paires de meules et de les faire reposer sur un beffroi. Elles sont mises en mouvement par un seul moteur au moyen d'un système d'engrenages en fonte. Son innovation sonne le glas du couple rouet/lanterne. Enfin, pour accroître le rendement, il met fin à la manutention manuelle en introduisant les élévateurs à godets et les vis d'Archimède pour le transport des produits depuis la livraison du grain jusqu'à l'exportation des farines. Dès lors, le traitement des céréales devient gravitaire et les équipements investissent les 
étages supérieurs. Souvent l'adoption de la "mouture à l'américaine" impose de rehausser les anciens moulins.

Ce procédé, élaboré entre 1780 et 1790 aux Etats-Unis, fait son apparition en Alsace vers 1840. Il est inauguré par l'un des moulins de Mulhouse, la Bleulatmühle ${ }^{11}$. Son introduction en Lorraine est contemporaine. En revanche, la majeure partie des moulins ruraux d'Alsace Bossue semble avoir ignoré cette révolution technique. Seuls les établissements d'une certaine envergure paraissent s'y être essayés, à l'image du moulin à céréales des frères De Langenhagen à Sarrewerden.

La mutation technique qui s'avère décisive pour le devenir des moulins d'Alsace Bossue ne s'opère qu'à la charnière des XIXe et XXe siècles avec l'introduction des broyeurs à cylindres ${ }^{12}$. Leur substitution aux meules permet de produire de plus belles farines et de mieux traiter les semoules. Certains établissements basculent alors dans la mécanisation complète de leur installation, mettant en application les principes édictés par Oliver Evans un siècle auparavant. Deux phases d'évolution technique sont ainsi fusionnées, les chaînes de fabrication nouvelles étant conçues à la lumière des innovations qui ont ponctué tout le XIXe siècle. De fait, l'apparition des élévateurs à godets en Alsace Bossue est souvent contemporaine de l'acquisition des premiers broyeurs à cylindres. L'espace intérieur du moulin est alors intégralement repensé et le grain suit un parcours de traitement complexe qu'on schématise à l'aide de diagrammes de mouture. Le moulin de Lorentzen connaît pareille évolution. En 1897, il se dote d'une nouvelle chaîne de fabrication déployée sur cinq niveaux. La force motrice est transmise au moyen de poulies et de courroies tandis que la manutention des produits est totalement mécanisée. Les moulins qui veulent survivre sont contraints de se moderniser. Ils sont nombreux à suivre cette voie entre 1890 et 1920, à l'image de ceux de Sarrewerden, Drulingen, Butten (moulin de Butten et Schleifmuehle), Waldhambach ${ }^{13}$ (Rehmuehle, Neumuehle), Voellerdingen ${ }^{14}$, et Bischtroff. Certains établissements, comme le moulin de Willer à Harskirchen ou celui de Sarre-Union, ont vraisemblablement connu deux phases successives de modernisation comme l'indiquent les différentes campagnes d'agrandissement. (fig. $n^{\circ}$ 9) 


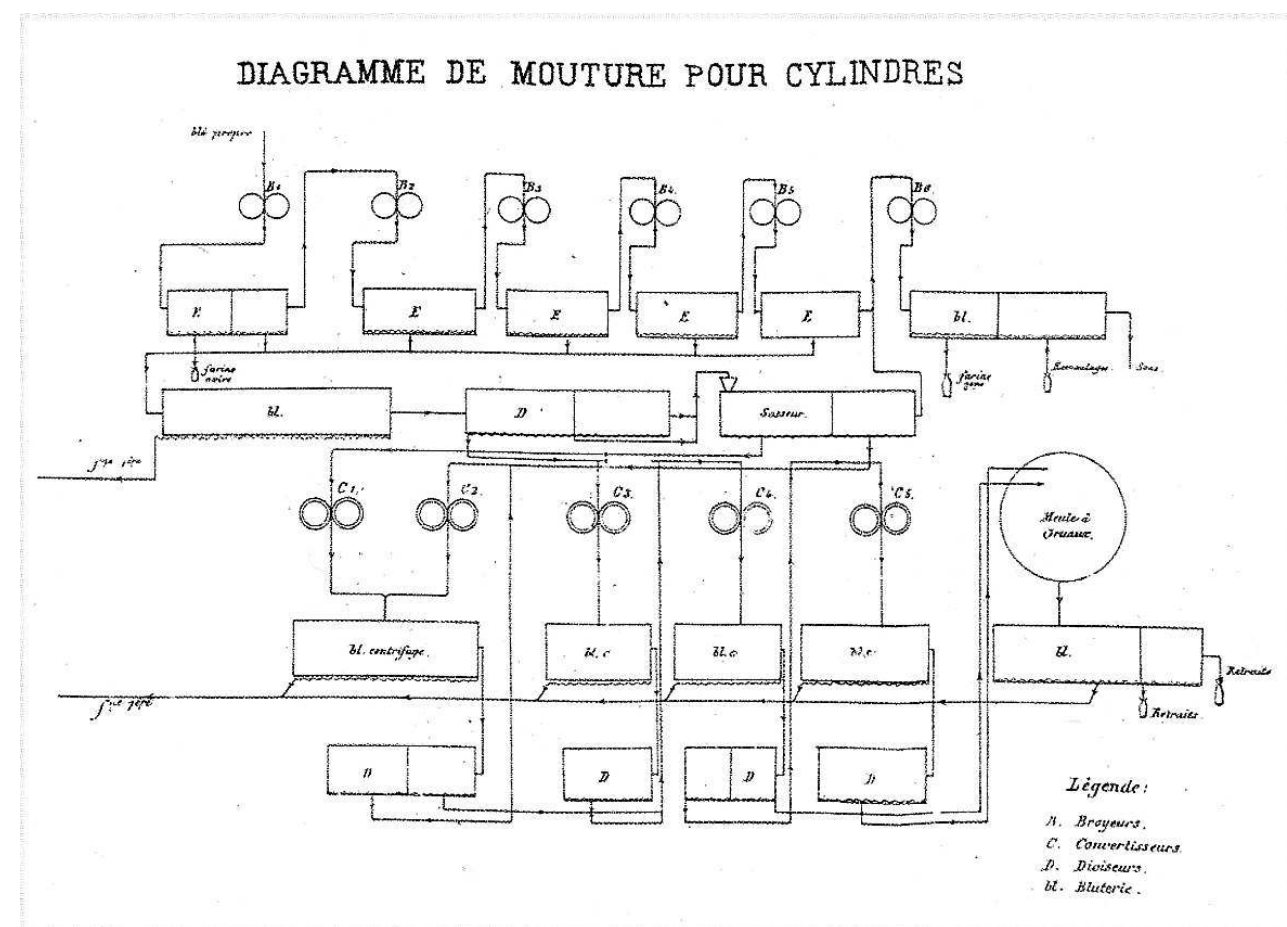

Diagramme de mouture pour cylindres, publié dans V. Pelsy, Histoire de la meunerie lorraine. Mémoires de l'Académie de Metz, 1896/1897. Repro. Inv. F. Schwarz

(c) Inventaire général, ADAGP, 2006

Ce mouvement d'intense rénovation s'accompagne de l'éclosion de véritables firmes spécialisées dans l'équipement des minoteries. L'entreprise Schneider et Jacquet de Strasbourg s'impose ainsi comme l'un des acteurs incontournables de ce marché. Nombreux sont les moulins d'Alsace Bossue qui ont recours à ses services. Il semble que la maison Kolb et Cie ait également procédé à l'installation de moulins à cylindres ${ }^{15}$. (fig. $\mathrm{n}$ $\left.{ }^{\circ} 10\right)$ 


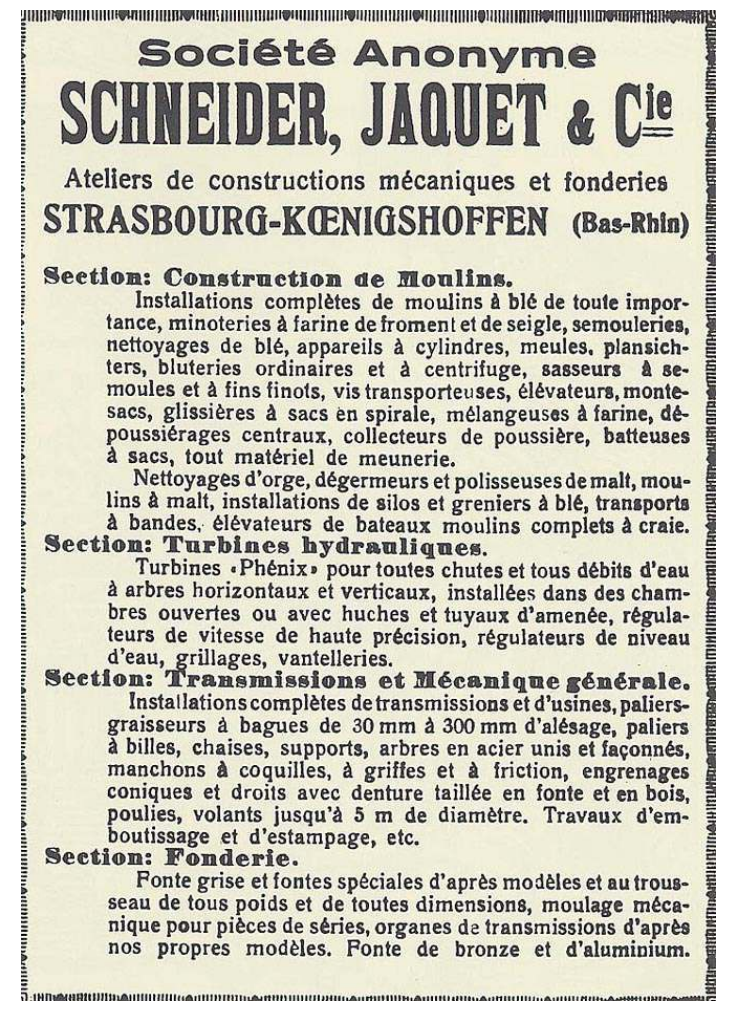

Encart publicitaire publié dans le Journal de la meunerie d'Alsace et de Lorraine et des régions limitrophes, 1924. Repro. Inv. F. Schwarz

(c) Inventaire général, ADAGP, 2006

$\mathrm{Au}$ cours du XXe siècle les perfectionnements techniques se poursuivent notamment sur le plan du blutage où le plansichter ${ }^{16}$ remplace progressivement les anciens blutoirs. Adopté dès le premier quart du XXe siècle au moulin de Drulingen, il se généralise vers 1950 dans les meuneries locales. De telles installations ont été répertoriées aux moulins de Bischtroff, Sarre-Union, Butten (moulin de Butten et Schleifmuehle), Waldhambach ${ }^{17}$ (Rehmuehle et Neumuehle) et Harskirchen (moulin de Willer) ${ }^{18}$.

\section{Une architecture peu différenciée}

16 Pendant longtemps les moulins d'Alsace Bossue ne présentent pas une architecture spécifique. Rien dans la mise en œuvre ne les différencie des constructions agricoles avec lesquelles ils voisinent. Les éléments caractéristiques tiennent alors à l'implantation et aux infrastructures hydrauliques déployées à leurs abords. Il faut attendre la seconde moitié du XIXe siècle pour que les mutations techniques affectant l'activité meunière génèrent une évolution morphologique des constructions. L'adoption de la "mouture à l'américaine" et son organisation gravitaire imposent le rehaussement des moulins traditionnels qui ne comportaient auparavant qu'un niveau de plain-pied coiffé d'un étage de comble. Nous avons vu que ce processus est tout à fait marginal à l'échelle de l'Alsace Bossue avant la fin du XIXe siècle. L'association un peu plus tardive de cette nouvelle technique aux broyeurs à cylindres impose cette tendance à la verticalité des 
édifices. Désormais, les moulins s'organisent sur quatre ou cinq niveaux. La chaîne de nettoyage des grains se déploie sur l'ensemble des étages, le broyage s'opère aux niveaux inférieurs tandis que le blutage s'effectue en partie haute. Les moulins de grande taille intègrent ces innovations en mettant à profit les niveaux supérieurs qui accueillaient jusque-là les trieurs ou servaient de greniers. Le moulin de Lorentzen est représentatif d'une telle évolution ${ }^{19}$. Au moment du réaménagement de l'établissement par l'entreprise Schneider et Jacquet en 1897, on installe des machines jusque dans les combles. Extérieurement, aucune transformation n'est observable. A l'intérieur, on relève simplement un renforcement de la structure porteuse des planchers par l'adjonction de poteaux et de sous-poutres métalliques profil IPN pour supporter le poids des nouvelles machines $^{20}$. Pour les moulins de plus petite taille, à l'image du moulin de Voellerdingen ${ }^{21}$ ou de celui de Willer à Harskirchen ${ }^{22}$, l'installation des broyeurs à cylindres et leurs accessoires se traduit par le rehaussement d'un ou deux étages. Le moulin de Sarre-Union ${ }^{23}$ connaît même deux phases successives d'agrandissement. (fig. $\mathrm{n}^{\circ} 11, \mathrm{n}^{\circ} 12$ )

Figure 11

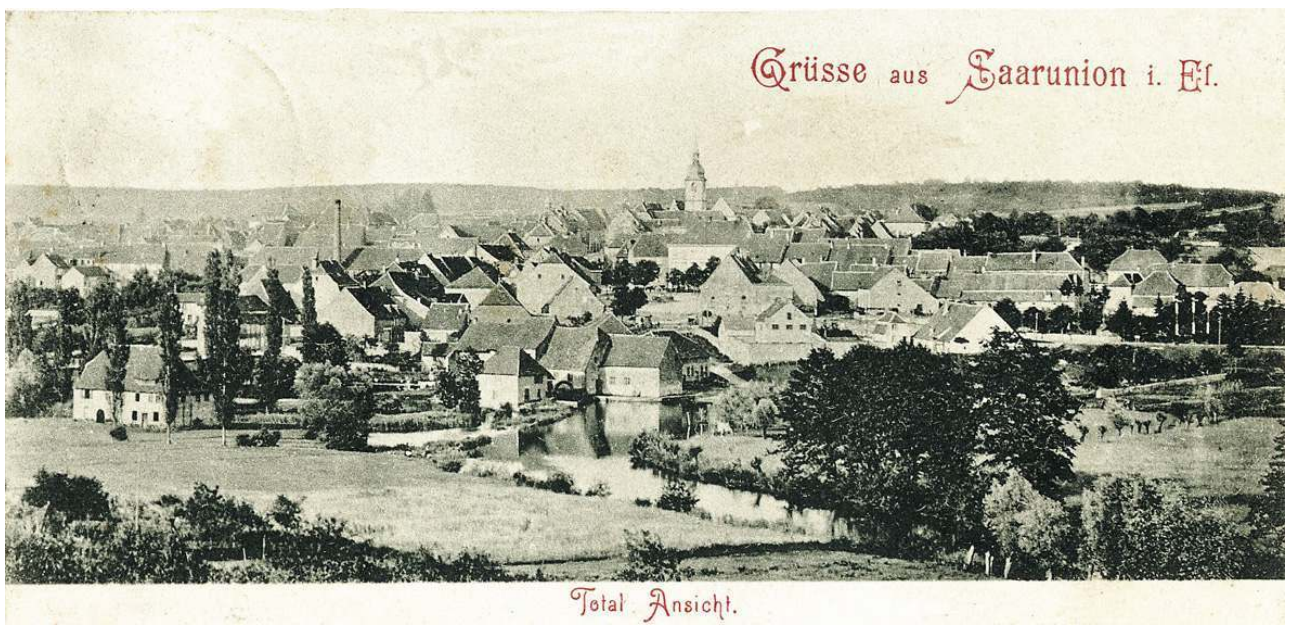

Le moulin de Sarre-Union vers 1900 (collection particulière). Repro. Inv. F. Schwarz

(c) Inventaire général, ADAGP, 2005 
Figure 12

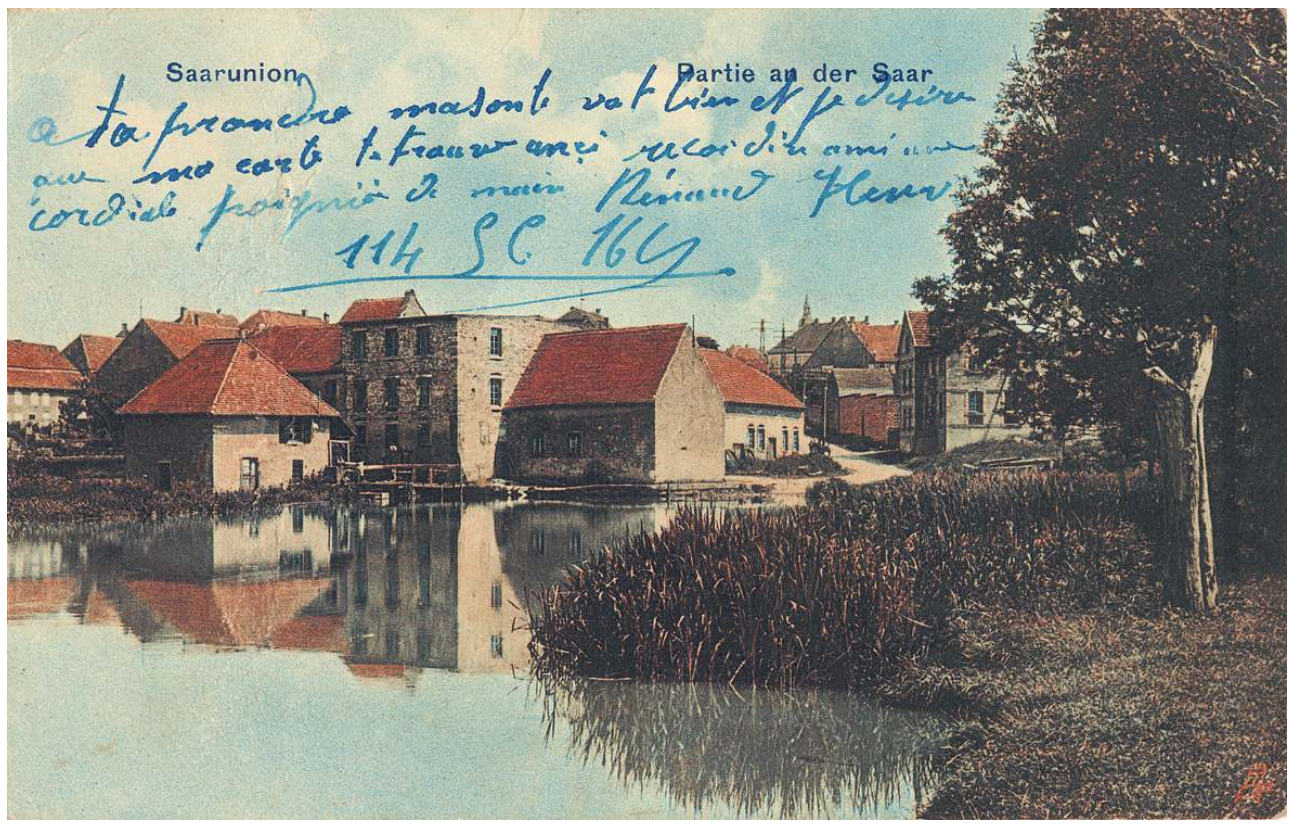

Le moulin de Sarre-Union vers 1914 (collection particulière). Repro. Inv. F. Schwarz

(c) Inventaire général, ADAGP, 2005

17 Cette évolution architecturale est donc intimement liée à la modernisation technique des moulins et dessine une nouvelle géographie qui oppose désormais petits moulins primitifs condamnés à disparaître et établissements engagés sur la voie de l'adaptation aux réalités du marché. (fig. $n^{\circ} 13$ ) 


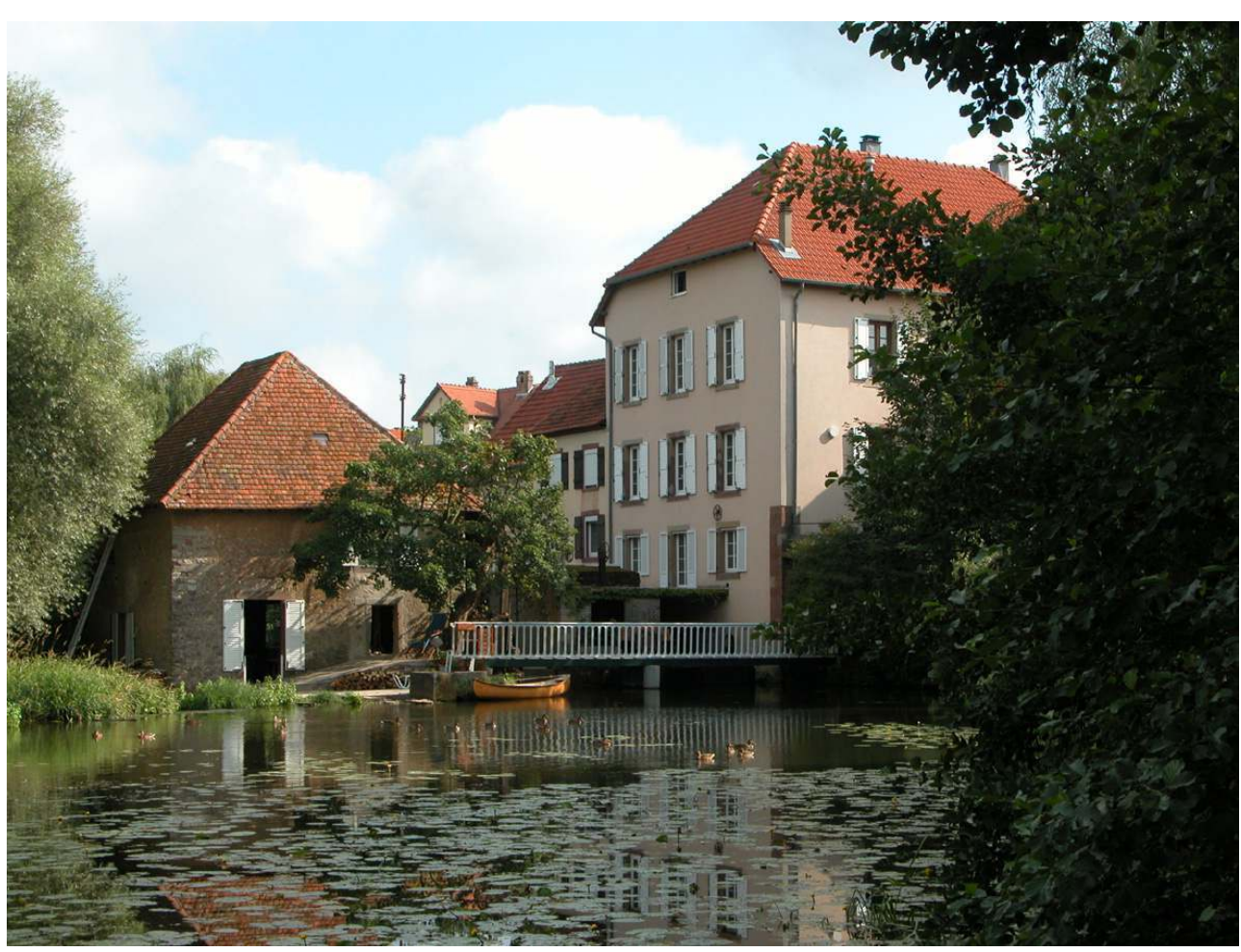

Le moulin de Sarre-Union aujourd'hui. Phot. Inv. F. Schwarz

(c) Inventaire général, ADAGP, 2005

\section{Une fidélité indéfectible à l'énergie hydraulique}

Parallèlement à leur mécanisation, les moulins d'Alsace Bossue s'adaptent aux mutations qui se font jour dans le domaine de l'énergie, manifestant toutefois une fidélité indéfectible à la force motrice de l'eau. Au début du XIXe siècle, l'ensemble des moulins du territoire fonctionne au moyen de roues hydrauliques verticales ${ }^{24}$. Il en va ainsi jusque vers 1890. La roue à aubes - aussi appelée roue en dessous parce que mue par un courant passant en dessous d'elle - est la plus répandue dans la région car elle ne nécessite pas de chute importante. Cela tient au relief peu accidenté de l'Alsace Bossue et à ses cours d'eau à pente modérée. Au moment de leurs remplacements périodiques, ces roues intègrent les perfectionnements qui sont le fruit de recherches et d'expérimentations incessantes. Sous l'impulsion de l'ingénieur Jean Victor Poncelet, on dote les roues en dessous d'aubes courbes pour en optimiser le rendement ${ }^{25}$. Cependant, pour accroître la puissance d'un moulin, plutôt que d'augmenter la taille de la roue, les meuniers préfèrent juxtaposer plusieurs roues motrices sur la même chute. On dénombre ainsi quatre roues au moulin de Lorentzen au milieu du XIXe siècle, trois aux moulins de Harskirchen ${ }^{26}$ (moulins de Willer et de la Honau ${ }^{27}$ ), de Butten, de Waldhambach (Rehmuehle ${ }^{28}$ et Kuppertsmuehle), de Voellerdingen, de Bischtroff et de Keskastel. On rencontre également, mais en nombre plus restreint, des roues en dessus, munies d'augets et utilisant le poids de l'eau. De telles installations réclament des chutes plus conséquentes. On en recense sur les cours de l'Eichel, de l'Isch et du Petersbach. (fig. n 14 ) 


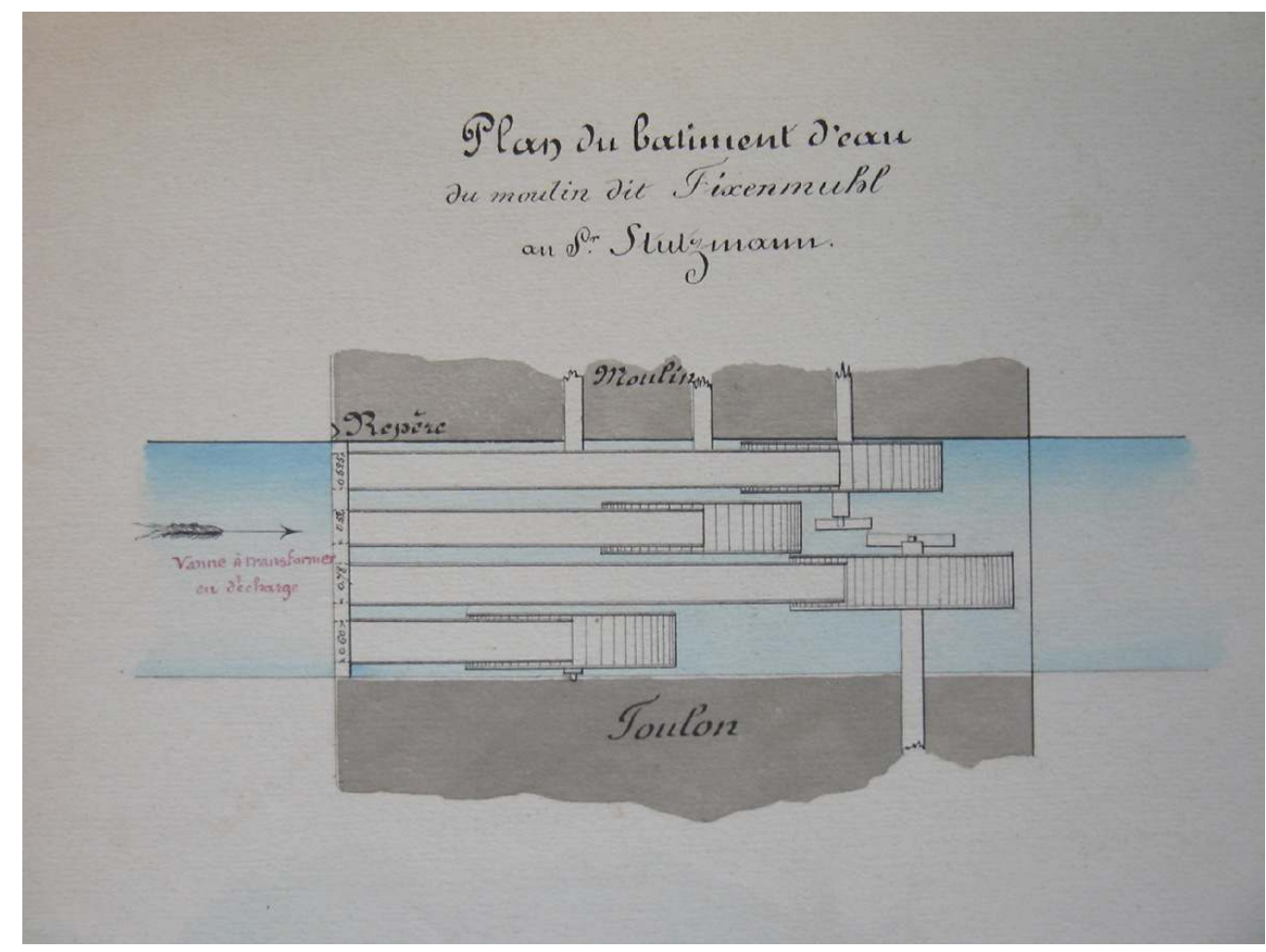

Plan du bâtiment d'eau du moulin de Fixenmuehle à Waldhambach. Auteur du plan : Ingénieur ordinaire Du Moulin. Document conservé aux Archives départementales sous la cote SP 1667/1. Repro. Inv. F. Schwarz

(C) Inventaire général, ADAGP, 2005. (Archives départementales Bas-Rhin)

19 Au moment où l'industrie s'émancipe progressivement de la contrainte liée à l'utilisation des cours d'eau en se tournant massivement vers l'énergie thermique, les moulins d'Alsace Bossue continuent de s'en remettre à l'énergie hydraulique. Le recours à la machine à vapeur apparaît comme tout à fait marginal. Seul le moulin du Bannholtz à Rimsdorf ${ }^{29}$ semble s'y convertir pour disposer d'une énergie de substitution, en cas de pénurie d'eau ${ }^{30}$. Or, en 1870 , vingt-huit moulins bas-rhinois se sont équipés d'une machine à vapeur ${ }^{31}$. Il faut attendre la diffusion de la turbine hydraulique pour que les établissements modernisent enfin leur installation motrice.

C'est vers 1880 que les premières commencent à équiper les moulins d'Alsace Bossue. Le moulin de la Kuppertsmuehle ${ }^{32}$, jumelé à une scierie, fait figure de précurseur. En 1881, il substitue des turbines hydrauliques aux anciennes roues, initiant de la sorte un mouvement qui se généralise au cours du premier tiers du XXe siècle. Le moulin de Drulingen franchit le pas en 1906, suivi de celui de Bischtroff en 1918. Un grand nombre encore en activité finit par s'en équiper entre 1926 et $1931^{33}$. (fig. $\mathrm{n}^{\circ} 15$ ) 
Figure 15

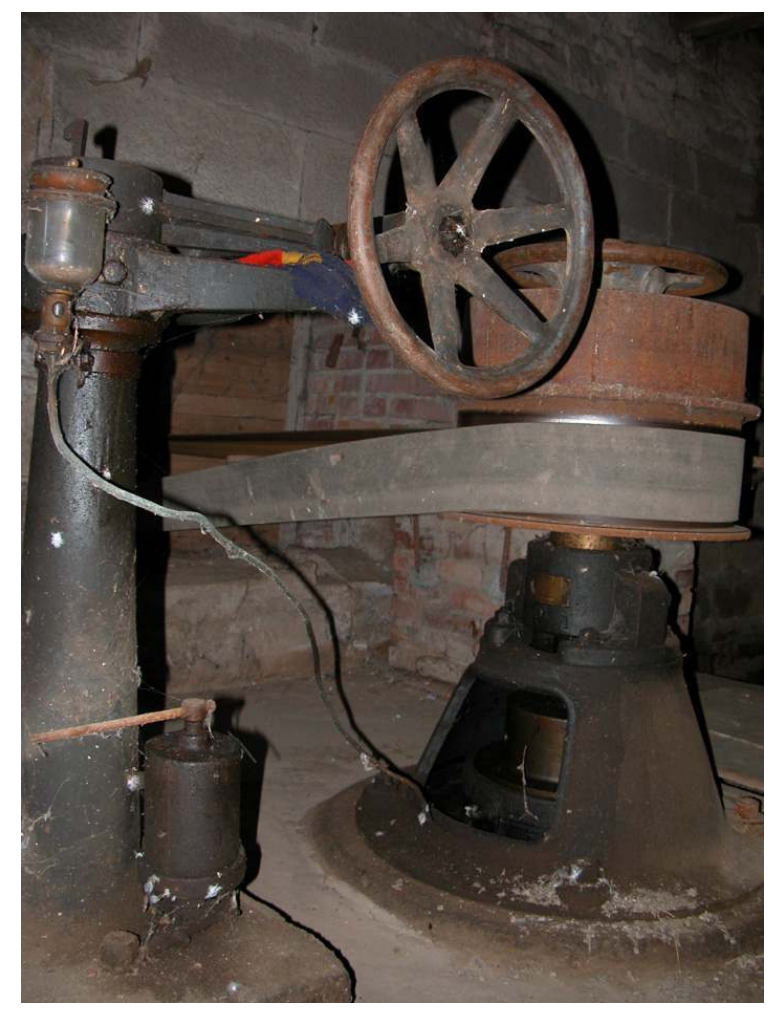

Turbine hydraulique du moulin de Rehmuehle à Waldhambach. Phot. Inv. F. Schwarz

(c) Inventaire général, ADAGP, 2005

21 Les autres conservent souvent leurs roues jusqu'à leur cessation d'activité. Au milieu du XXe siècle, certains établissements optent pour des roues à structure métallique intégrale, confectionnées par des artisans locaux. Les moulins de Sarre-Union, Voellerdingen et celui de la Honau à Harskirchen suivent cette voie. Une dernière étape est franchie lorsque les moulins encore en activité renoncent à l'énergie hydraulique au profit de l'électricité, plus souple d'utilisation. C'est le choix fait par le moulin de Voellerdingen, en 1971. De nos jours encore, sept roues hydrauliques s'offrent à la curiosité du promeneur à travers l'Alsace Bossue ${ }^{34}$. L'une d'entre elles continue d'actionner le moulin de Willer à Harskirchen, faisant le lien entre le passé et le présent.

\section{Conclusion}

La particularité des moulins d'Alsace Bossue, au plan régional, réside dans le relatif retard de développement technique qui a marqué leur histoire. Il en est résulté une permanence plus marquée qu'ailleurs des mécanismes ancestraux et d'une architecture sans grande spécificité. Par la suite, la mécanisation des chaînes de fabrication a été progressive, ce qui explique l'association, au sein d'un même établissement, de machines d'époques différentes. Deux de ces moulins, encore en activité, constituent de la sorte un formidable support pédagogique pour appréhender les innovations techniques qui ont scandé l'histoire de la meunerie et pour saisir la complexité du processus d'élaboration de la farine. 


\section{NOTES}

1. Le présent exposé porte sur le territoire de l'Alsace Bossue circonscrit aux limites administratives des cantons de Drulingen et de Sarre-Union. Il ne prend pas en considération sa portion plus orientale qui s'étend vers le canton de la Petite-Pierre.

2. Les cantons de Drulingen et de Sarre-Union ont fait l'objet, de la part du Service régional d'Inventaire d'Alsace, d'une enquête thématique consacrée au patrimoine industriel au cours de l'année 2005. Dans ce cadre, Chip Buchheit a inventorié les vestiges industriels qui n'étaient pas liés à l'activité de meunerie ainsi que la minoterie Schlumberger de Wolfskirchen.

3. GRAD, Charles. Etudes statistiques sur l'industrie de l'Alsace. Strasbourg : 1879, p. 445.

4. Voir dans la base Mérimée : notice IA67005723.

5. PELSY, Valentin. Histoire de la meunerie lorraine. Mémoires de l'Académie de Metz, 1896/1897, p. 277.

6. Voir dans la base Palissy : notice IM67017207.

7. Bluterie composée de tamis horizontaux enfermés dans une caisse parallélépipédique montée sur un excentrique qu'actionne une transmission par courroie. Les tamis sont ainsi animés par un vif mouvement oscillatoire et circulaire.

8. PELSY, Valentin. Histoire de la meunerie lorraine. Mémoires de l'Académie de Metz, 1896/1897, p. 220.

9. Ce procédé est également connu sous le nom de “mouture à l'anglaise" car les Anglais, qui disposaient de la maîtrise de la fabrication de la fonte dès le XVIIIe siècle, furent les propagateurs de la nouvelle technique.

10. DAUMAS, Maurice. L'archéologie industrielle en France. Paris : 1980, p. 220-221.

11. Histoire documentaire de l'industrie de Mulhouse et environs au XIXème siècle. Mulhouse : 1902, p. 753.

12. Albert Brunner, meunier à Cernay, introduit les premiers cylindres en Alsace en 1878 (Voir Histoire documentaire de l'industrie de Mulhouse et environs au XIXème siècle. Mulhouse : 1902, p. 758).

13. Voir dans la base Palissy : notice IM67017208.

14. Voir dans la base Palissy : notice IM67017221.

15. PELSY, Valentin. Histoire de la meunerie lorraine. Mémoires de l'Académie de Metz, 1896/1897, p. 263.

16. Il s'agit d'une bluterie plane mise au point en 1887, en Hongrie, par un meunier de Budapest, Hagenmacher. Elle est introduite en France dès 1889 par la maison de constructions BraultTeisset et Gillet, de Chartres.

17. Voir dans la base Palissy : notice IM67017208.

18. Voir dans la base Palissy : notice IM67017207.

19. C'est le cas également des moulins de Schleifmuehle à Butten, de Rehmuehle et Neumuehle à Waldhambach.

20. BRUA, Jean-Christophe. Etude sur le fonctionnement de l'ancien moulin de Lorentzen. 2005, p. 38.

21. Voir dans la base Mérimée : notice IA67006034.

22. Voir dans la base Mérimée : notice IA67005808.

23. Voir dans la base Mérimée : notice IA67005955.

24. Etat des moulins à farine actuellement en activité dans l'arrondissement de Saverne du 14 février 1809 (Archives départementales du Bas-Rhin, 11 M 34). 
25. DESCOMBES, René. La Sarre au fil de l'eau. Sarrebourg : 1982, p. 148.

26. Voir dans la base Mérimée : notice IA67005808.

27. Voir dans la base Mérimée : notice IA67005809.

28. Voir dans la base Mérimée : notice IA67000200.

29. Voir dans la base Mérimée : notice IA67006052.

30. Une autorisation d'installer une machine à vapeur est délivrée en 1864 au meunier Nicolas Henn. Le moulin est détruit par un incendie en 1882. Dans l'état actuel de nos recherches, il n'a pas été possible de déterminer si cette installation a bien été réalisée.

31. ACUNA, Leonardo. Les machines à vapeur dans le Bas-Rhin : 1851-1870. Mémoire de maîtrise, USHS, Strasbourg : 1990, p. 63.

32. Voir dans la base Mérimée : notice IA67007851.

33. Il s'agit du moulin de Schleifmuehle à Butten et du moulin de Sarrewerden (1926), du moulin de Rehmuehle à Waldhambach (1928), du moulin de Butten (1931) et du moulin de Neumuehle à Waldhambach

34. Outre celle du moulin de Willer à Harskirchen, on les trouve à Sarre-Union (deux roues métalliques à aubes incurvées), à Voellerdingen (roue métallique à augets), à Lorentzen (roue à axe métallique et structure bois à aubes droites, vestiges) et à Harskirchen, au moulin de la Honau (deux roues métalliques à aubes incurvées).

\section{RÉSUMÉS}

Le corpus des moulins d'Alsace Bossue est caractérisé par un processus de concentration limité et un développement technique tardif en raison de l'éloignement des grands centres urbains et des voies de circulation majeures. De cet état de fait découlent les spécificités mises en évidence : une mécanisation lente, une architecture spécifique qui ne s'affirme véritablement qu'au XXe siècle et un attachement jamais démenti à l'énergie hydraulique jusqu'à une période très récente. Cet enclavement relatif et l'orientation majoritairement agricole de ce territoire expliquent aussi le maintien en activité et ce jusqu'à nos jours, de moulins ruraux sur ces terres situées aux confins de l'Alsace.

The corpus of water mills studied in the region known as the Alsace Bossue is characterised by their limited concentration and their late technical development, to be explained by this region's distance from major urban centres and from transport networks. This situation explains some of the particularities to which a recent inventory was able to draw attention: the slow process of mechanisation, specific architectural forms which reached maturity only during the 20th century and an unfailing attachment to water power, even up to recent times. The region's relative isolation and its primarily agricultural vocation explain how some of these rural water mills at the frontiers of Alsace are still in use today. 


\section{AUTEUR}

FRANCK SCHWARZ

Chargé d'études pour le patrimoine industriel auprès du Service chargé de l'inventaire général du patrimoine culturel, Région Alsace. frank.schwarz@region-alsace.eu 